\title{
The Impact of MERS-Cov: Service and Systems Re-design: The Creation of the Caswell - Hijazi Model
}

\author{
Angela Caswell ${ }^{1}$, Raed Hijazi ${ }^{2}$ \\ ${ }^{1}$ Department of Nursing King Abdullah Specialist and Children's Hospital, Saudi Arabia Kingdom, University of South Wales and South Bank \\ University, United Kingdom \\ ${ }^{2}$ Department of Emergency, Shaikh Khalifa Medical City, Abu Dhabi, George Washington University, Columbia, King Abdulaziz University, \\ Saudi Arabia Kingdom, Seton Hall University, New Jersey
}

Email address:

atcaswell@gmx.com (A. Caswell),hijazimd@yahoo.com (R. Hijazi)

\section{To cite this article:}

Angela Caswell, Raed Hijazi. The Impact of MERS-Cov: Service and Systems Re-design: The Creation of the Caswell - Hijazi Model. American Journal of Nursing Science. Vol. 8, No. 5, 2019, pp. 200-209. doi: 10.11648/j.ajns.20190805.11

Received: May 11, 2019; Accepted: July 5, 2019; Published: August 5, 2019

\begin{abstract}
Middle Eastern Corona virus (MERS-Cov) has been acknowledged globally as a novel and evolving pathogen. First detected in 2012, approximately 1,000 confirmed cases reported by World Health Organization and linked to travel to Saudi Arabia. The declaration of an epidemic in the Arabian Peninsula gained the global attention. The summer of 2015 witnessed a MERS-Cov outbreak resulting in the closure of a major Middle Eastern university teaching hospital. Overcrowding and delays were acknowledged as contributory factors. Patient flow processes were not streamlined resulting in frustration amongst staff and patients. Lack of knowledge related to the mode of transmission of this pathogen added to the challenges faced within the Emergency Department. A complete system and service re-design took place with the introduction of the Kingdom's first Drive Through Screening and Streaming Unit (along with secondary screening and surveillance checkpoints) using an Acute Respiratory Illness tool, to direct potentially infected patients to designated isolation areas to a flu clinic equipped to manage all suspected cases of MERS-Cov and isolated away from the main Emergency Department. This novel concept has been developed to ensure safe and efficient screening and streaming of suspected cases, The Caswell - Hijazi Model.
\end{abstract}

Keywords: MERS-Cov, Service and System Redesign, Caswell - Hijazi Model

\section{Introduction}

Overcrowding, poor compliance with basic infection, prevention guidelines and lack of knowledge about the evolving, novel pathogen led to the outbreak and closure of one of the largest military university teaching hospitals in the Middle East.

During the frenetic days of the MERS-Cov outbreak, the organization was unable to identify or contain the spread of the virus. The ensuing days became untenable as teams became isolated to divisions in an attempt to control further spread of the virus. The Emergency Room was especially affected as two members of the nursing and medical team contracted the virus leading to admission to intensive care. Isolation, segregation and uncertainty added to the tensions within the hospital as well as the local community.

Emergency room staff was no longer authorized to travel on the campus trams that linked to the university campus.
Local buses were unhappy about staff boarding and many colleagues refused to sit next to them or share accommodation.

Staff who had arranged to travel home during their vacation time had also experienced serious challenges. Staff arriving at global destinations had been placed in quarantine. Many staff placed on a no fly list during a nationally agreed lock down period. The implications were personal, professional and economic.

\section{Problem}

Overcrowding, poor compliance with basic infection, prevention guidelines and lack of knowledge about the evolving, novel pathogen led to the outbreak and closure of one of the largest military university teaching hospitals in the Middle East. 


\section{Background}

During the frenetic days of the MERS-Cov outbreak, the organization was unable to identify or contain the spread of the virus. The ensuing days became untenable as teams became isolated to divisions in an attempt to control further spread of the virus. The Emergency Room was especially affected as two members of the nursing and medical team contracted the virus leading to admission to intensive care. Isolation, segregation and uncertainty added to the tensions within the hospital as well as the local community.

Emergency room staff was no longer authorized to travel on the campus trams that linked to the university campus. Local buses were unhappy about staff boarding and many colleagues refused to sit next to them or share accommodation.

Staff who had arranged to travel home during their vacation time had also experienced serious challenges Zaid et al [1]. Staff arriving at global destinations had been placed in quarantine. Many staff placed on a no fly list during a nationally agreed lock down period. The implications were personal, professional and economic.

Unfortunately little reliable data had been collected prior to the outbreak. The only data presented relied upon crude statistics related to numbers and subcategories of patients who attended the department. There was no evidence of measurable elements such as length of stay, repeat attendances or delays.

Therefore this project commenced at ground zero with a fresh start at data collection and analysis that was benchmarked against international performance indicators.

\section{Method}

Utilizing Plan-Do-Study-Act and lean quality model, the effectiveness of the Drive-through Screening Unit (DTSU) and Rapid Assessment and Management (RAM) were measured. A mixed methodology of qualitative and quantitative data was analyzed over a 12 month period following the introduction of the revised system. An Ishikawa chart was used to examine cause and effect. The variables were plotted using a Pareto chart. Clear goals, purposes, strategies and objectives were established to set against a definitive timeframe mapped using a Gantt chart. The patient's journey through the department was process-mapped using a series of scenario- based flow processes as part of (PDSA) cycles over the pre and post-opening period.

Measurement of performance, surveillance, containment, control success was examined and refined to prevent further outbreaks. Employ a strategy to optimise sustainability through empowering staff through education, engagement, mastery and autonomy using the DICE Theory Sirkin et al 2005 [2]. The DICE Theory enables teams to calculate the likelihood of success of their project, using a simple formula to calculate: Duration $=1$, Integrity $=1$, Senior Commitment $=1$, Low Level Commitment $=1$, Effort $=3$. D.I.C.E. $=D=(1 x 1)=$ $(2 x C 1)=C 2=E=7$. The overall score range $7-14$ places the team in the 'win' zone with high probability of success.
The application of this score is a leading indicator of the likelihood of success and the sustainability of a project based on the duration, integrity and team performance, commitment and effort required. The DICE Score Card provides a calculation of the probability of success of a project based upon a pre-determined number of variables. The 'ownership' of the system re-design will be illustrated in the key performance indicators along with the infection control reports of zero transmission.

A robust and structured infection prevention and control training program was developed and implemented for all staff to ensure understanding of and compliance with infection prevention and control (IPC) guidelines.

The average annual census of 219,000 patients reflected a slow initial start following the re-opening of the department. Patients screened via the 'Drive-through Unit' 144,717 with a mean time of 22.49 seconds demonstrating no delays and ensuring the patient was directed to the target area for assessment and isolation. Secondary screening and surveillance checkpoints were established at two strategic areas within the department were established to ensure a robust process to prevent potentially infected patients from entering the main stream of the department. Identified patients were directed to negative pressures rooms within the department.

The mean LOS (length of stay) for the Flu Clinic was 1.94 hours. This was benchmarked against international standard time of 4 hours. Rapid Assessment and Management Unit (RAM) had a mean LOS of 27 minutes. This was compared to international average waiting time of 47.4 minutes McCaig and Nawar 2006 [3].

Used to undertake an organisational gap analysis Nadler-Tuchman, 1977 [4]. Results identified key themes as potential contributory factors to the sentinel event that led to the hospital closure. Applying a lean methodology to patient flow management was essential to reducing overcrowding in the ED and successfully screening and isolating suspected cases from the main stream of the department David et al, 2008 [5]. Organisational risk assessment was a fundamental requirement to preventing a recurrence of the event.

A mixed methodology was applied through a series of Gap analysis, 4 PDSA cycles and lean. Key organizational factors were identified as contributing to the failure. Performance data for 4 divisible calendar quarters from November 2015 to October 2016 was collected. The hospital had closed during August 2015 and reopened two months later; no previous data was available against which to measure. Lean methodology was applied to streamline the process of safe and efficient assessment, management and care to avoid unnecessary delays. Daily multidisciplinary team meetings were held during which key performance indicators were analyzed in detail from the previous day activity to identify delays and challenges has encountered. Surveillance of infection prevention and control (IPC) compliance and patient flow was meticulously adhered to. Every member of staff acted as surveillance compliance monitors, correcting any flaws in the adherence to strict IPC guidelines. 


\section{The Nadler-Tuchman Model}

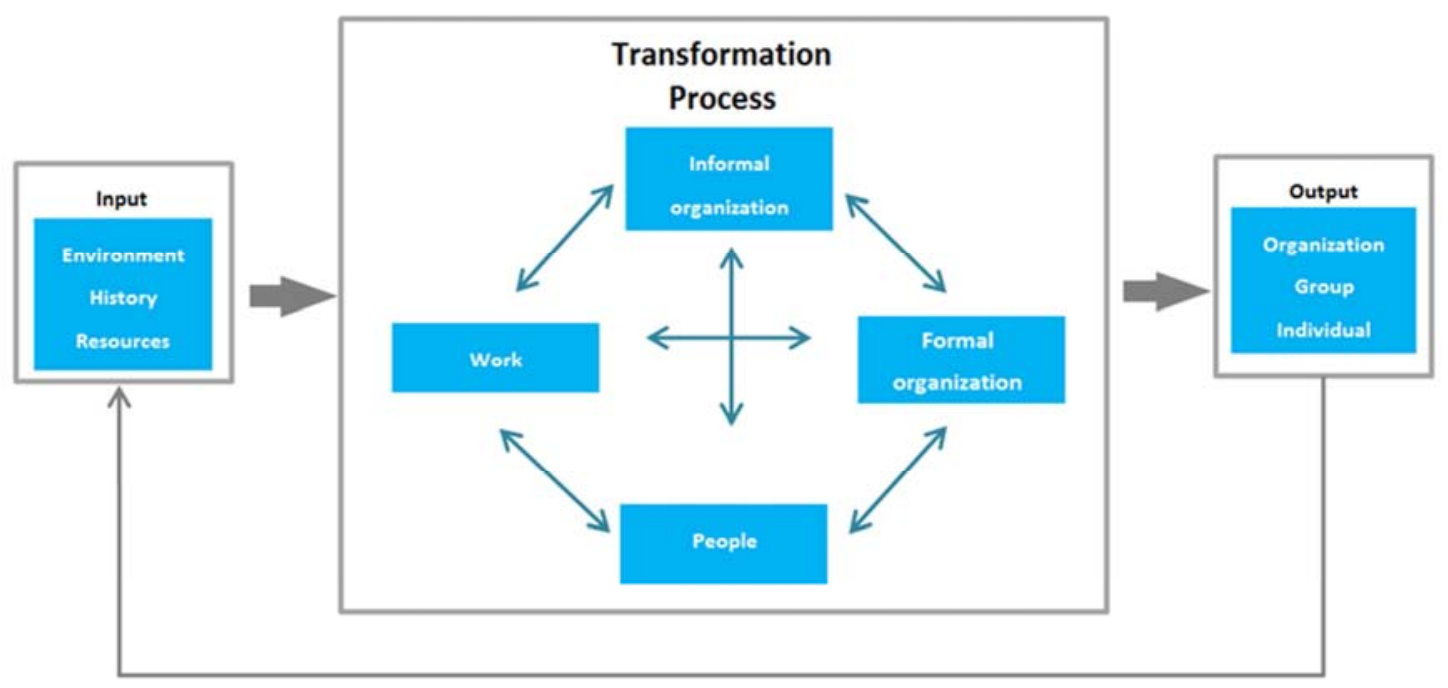

Figure 1. The Nadler-Tuchman Model.

An infection control practitioner was assigned to the department $24 / 7$ to be available for immediate consultation and action.

Organizational Risk Management

The efficiency of the ER relies upon speed and the ability to efficiently manage high numbers of patients, essential to cope with daily attendance. There is a direct correlation between increased length of stay of patients in ER due to overcrowding and increased mortality Sahin et al 2015 [6]. This was supported by Armony et al 2015 [7] from a queueing-science perspective which examined the essential synergy between emergency admissions and available hospital beds. Effective management of capacity resource is essential to supporting flow within the

Patient Flow Methodology a) Creation of bed management team working in close collaboration with all specialties and located within the ER itself.

b) The push/pull methodology was strictly applied. This resulted in almost zero boarding patients in the department awaiting inpatient beds more than 6 hours from time of arrival (benchmarked against international standards of best practice and evidence based clinical outcomes linked to optimal outcomes versus increased length of stay in ER; Singer et al 2011 [8] using a retrospective cohort study of 41,256 admissions. The study showed that boarded patients $<2$ hours had a mortality rating for severely acute patients of $2.5 \%$ compared to those of $>12$ hours increased to $4.5 \%$ $(\mathrm{p}<0.001)$.

\section{Results}

NO. OF PTS. THRU DTU

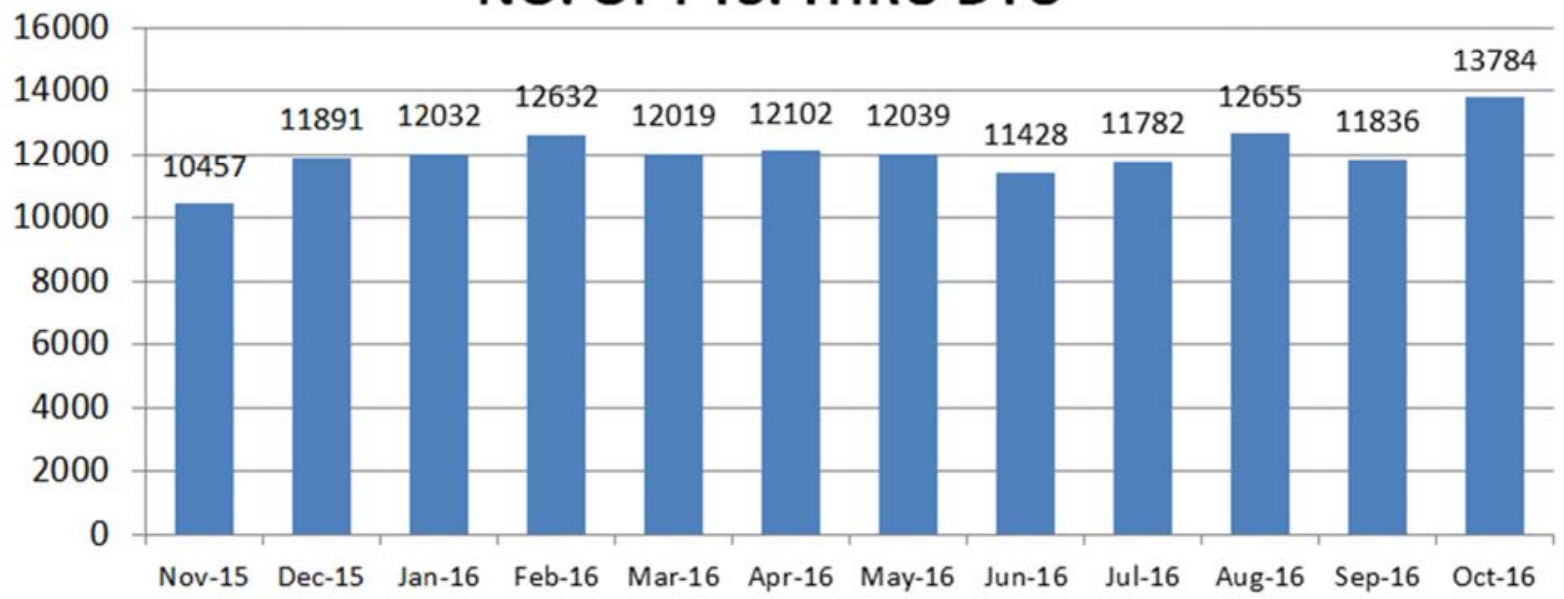

Figure 2. The New Drive Through Unit: Performance Measured in Seconds. 


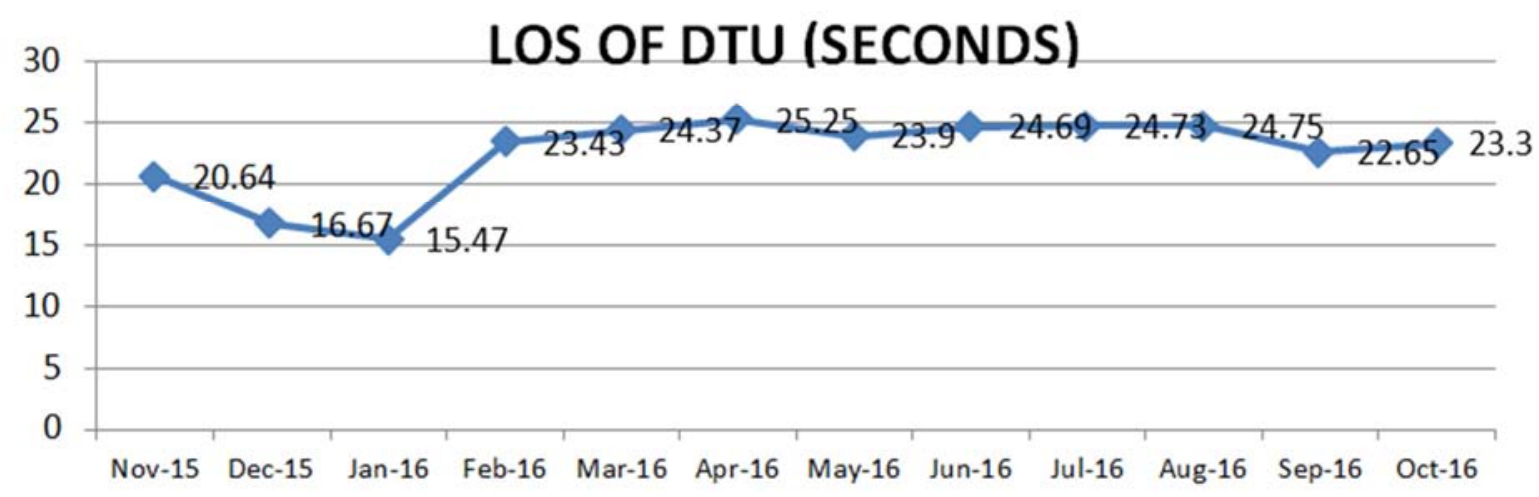

Figure 3. Length of Stay in Drive Through Unit: Performance Measured in Seconds.

The Volume of Patients Progressively Increasing: Performance however improving.

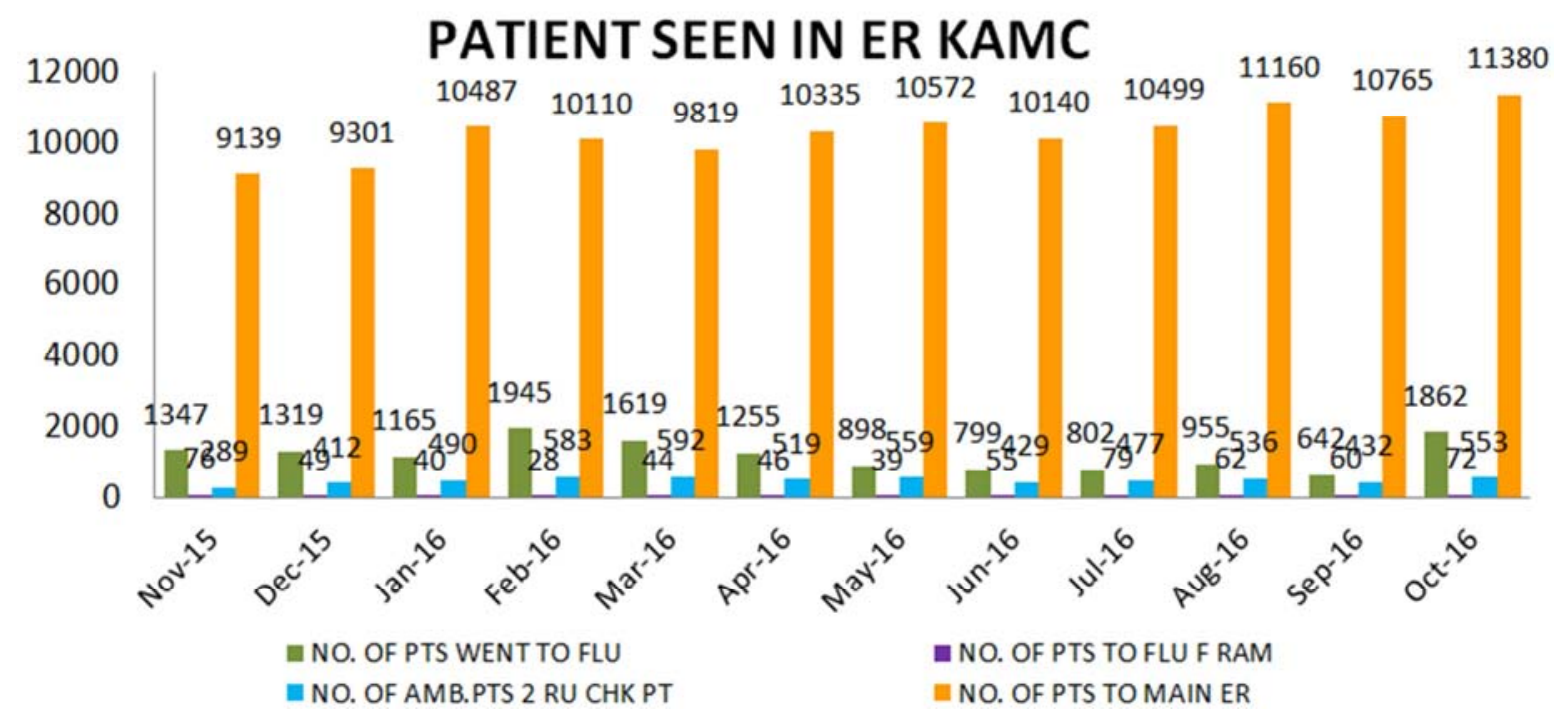

Figure 4. Total Number of Patients Seen in ER KAMC.

Length of Stay in the Flu Unit Measured in Hours: achieving a mean time of 2.01 hours total LOS during phase 4 . International benchmark is 6 hours.

All patients triaged using the Canadian Triage Acuity Scale CTAS Beveridge et al 1998 [9].

Level 1-immediate

Level 2-emergent (time to physician $<15$ minutes)
Level 3- urgent (time to physician $<30$ minutes) Level 4- less urgent (time to physician $<1$ hour) Level 5- non-urgent (time to physician $<2$ hours) N.B. with the introduction of the rapid assessment model, the department consistently out- performed these formulated waiting times to achieve a world- class flow methodology.

FLU LOS

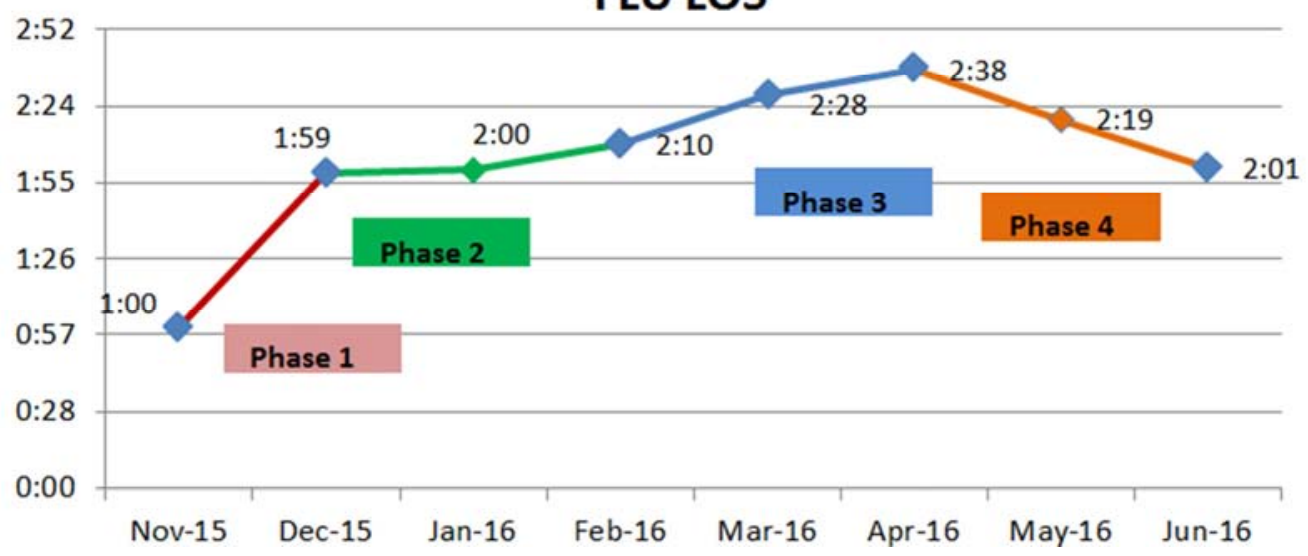

Figure 5. Length of Stay in Flu Unit: Performance Measured in Hours. 
Creation of New Units to Reduce Demands on Inpatient Beds

To support the patient organizational flow model, two new units were established as a sub-section of the ER. The Clinical Decision Unit and the Acute Medical Unit.

Scott et al 2009 [10] undertook a synthesis of data of nine peer-reviewed reports of before-after analyses of seven units introduced into the UK and Ireland were analysed. Two studies, one prospective, reported significant reductions in in-patient mortality between 0.6 and $5.6 \%$ points following commencement of AMU. Four studies reported significant reductions in the length of stay between 1.5 and 2.5 days. Waiting times for patient transfer from emergency departments to medical beds decreased by $30 \%$ in one study. In three studies, the proportion of medical patients discharged directly home from the AMU increased by $8-25 \%$ points. Three studies noted no increase in 30-day readmission rates following unit commencement. Two studies described significant improvements in patient and staff satisfaction with care. Eight non-peer-reviewed reports relating to 48 units confirmed reductions in the length of stay.

Hassan 2003 [11] described the concept of a CDU as old but with a paradigm shift refinement making it the organizational gatekeeper. Many organizations use a standard time frame of between 24-48 hours during this time of observation to fasttrack investigations and treatments using clear protocols for specific patient groups for whom this unit would be sui Figure. The internationally benchmarked discharge rate from these units is $85 \%$ Pearson et al 1995 [12].

The ER decided to establish 6 beds for CDU with a time frame of 12-16 hours with an average discharge rate of $73 \%$. The AMU with timeframes of up to 72 hours with a discharge rate of $77 \%$. Whilst these units are slightly below international benchmarked rates, there is a significant improvement with overall reduction in admissions.

\section{CDU LOS}
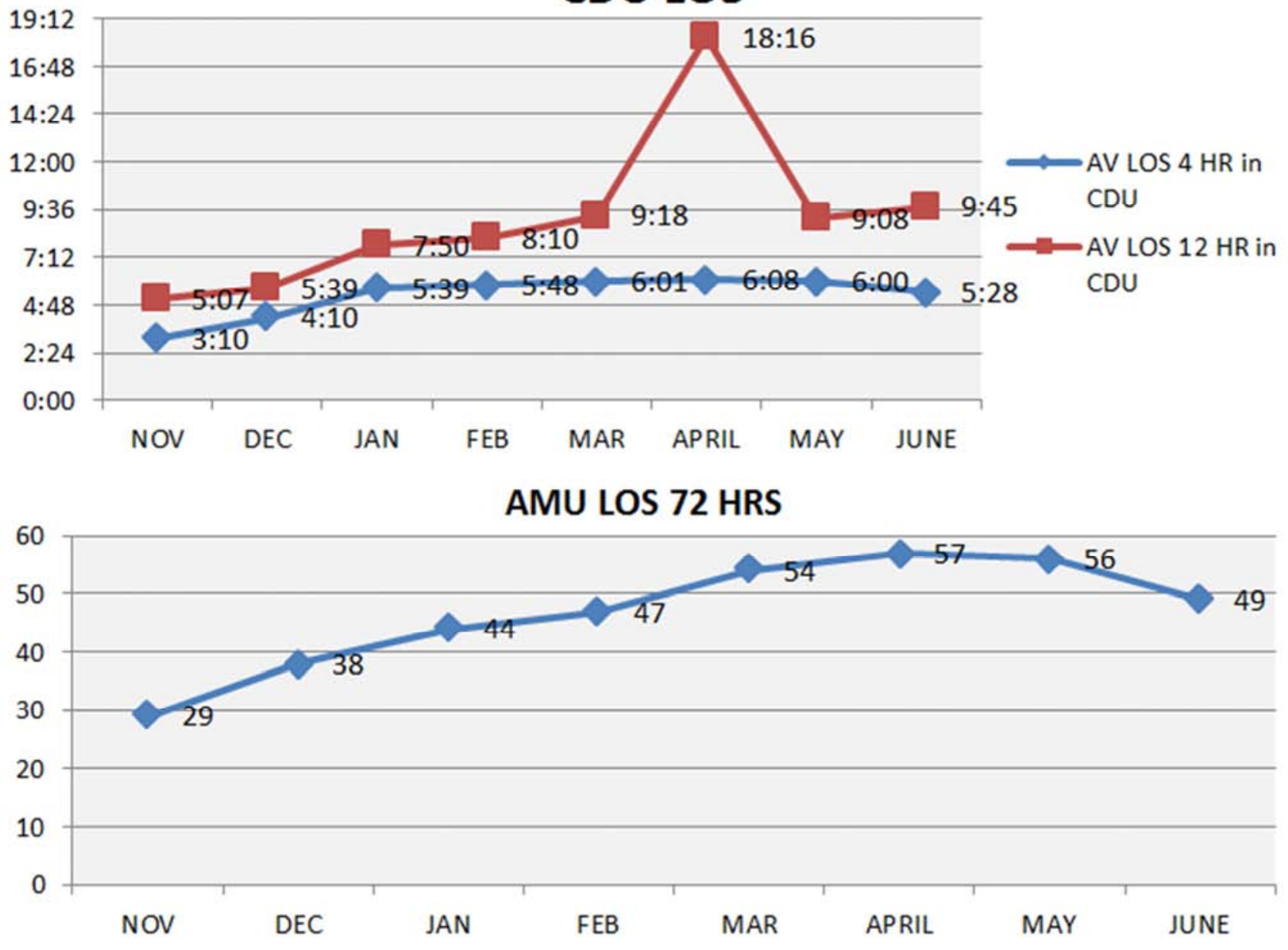

Figure 6. Length of Stay in $C D U$ and $A M U$.

\section{The Human Factor}

The next stage of this overarching analysis will examine the effects on individuals during the sentinel event that led to the closure of the hospital. How it impacted on their personal as well as professional lives. This qualitative element of the work will be undertaken in July 2017 following the ethical approval of the organization and the personal consent of key stakeholders who will be invited to participate in a series of short interviews.

Lessons and limitations 
Some limitations that impacted on the study were related to lack of measurable data prior to the hospital closure. Lack of knowledge among all staff of international benchmarked performance standards, against which to measure outcomes.

This was a steep organizational learning curve that was forced to move forward at a breakneck pace in order to achieve a rapid and sustainable recovery phase. Globally, continued surveillance was evident from international organizations such as World Health Organization, Council for Communicable Diseases, Joint International Commission America and nationally, Ministry of Health.

Declaration of interests

The authors declare that they have no competing interests.

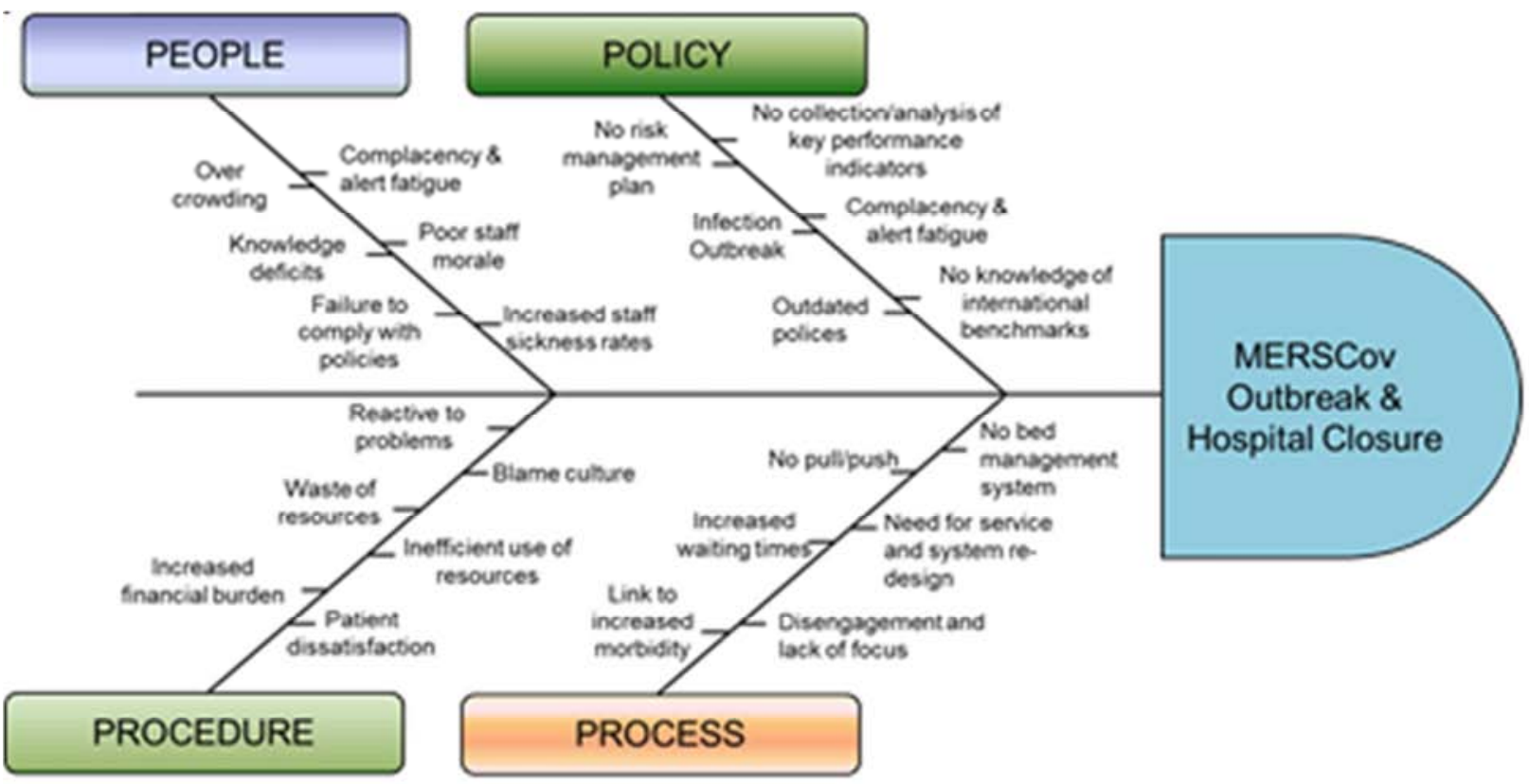

Figure 7. Root Cause Analysis using the Ishikawa Diagram.

\section{Conclusion}

A paradigm shift in organizational awareness, and risk assessment that led to an internationally acknowledged methodology that has shaped systems and service redesign with evidence to support its effectiveness and sustainability. The service and systems re-design has effectively demonstrated that the measured key performance indicators (KPI's) out- performed international benchmarked standards resulting in zero delays and zero transmission of infection. In spite of high volume of attendances, the Emergency Department has successfully sustained impressive KPI's that facilitate safe and efficient patient assessment, management and flow. With the implementation of a structured bed management service, the pull/push method of organizational patient flow has meant almost no boarded patients remain in the Emergency Department. There has been zero hospital acquired cases of MERS-Cov since the introduction of the new process. A recent patient satisfaction survey yielded an overall rating of excellent.

Members of the multidisciplinary teams were selected to achieve a balanced cross -representation from all staff in ED.
This included infection control staff, paramedics and housekeeping supervisors. The objective was to honestly identify the causes of the outbreak and the effects not only upon the department but the hospital as a whole. This collaborative meeting was undertaken daily.

The next stage was a multi-voting exercise wherein the weighting of each cause and effect potential was assigned a percentage representing what the team considered the most serious risk and the related effects. A Pareto chart was developed based upon this session. Pareto theorized that, on balance, $20 \%$ of the underlying causes resulted in $80 \%$ of the resulting outcomes. (Figure 8) illustrates the Pareto chart ranking the identified causes in order of priority.

Outcome measures were determined to identify how the system was performing equating to the ultimate result. Process measures were examined to determine which steps in the process were obtaining the optimal results as planned to affect the outcome measures. Balancing measures which were often not directly related to the aim but to support the team in assessing whether the changes designated to improve one part of the system may in fact be introducing more problems Scoville and Little, 2014 [13]. 


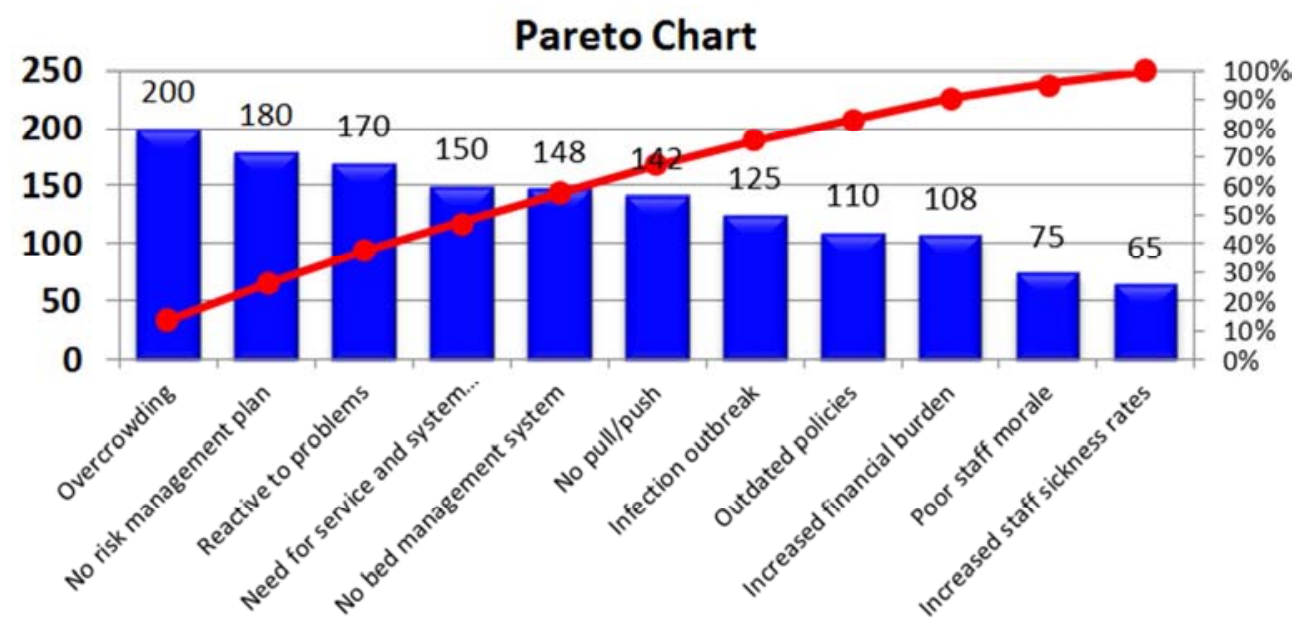

Figure 8. Pareto chart ranking the identified causes in order of priority.

The red line graph pegged to the right vertical axis represents the cumulative percentage of the voting related to the significance rating of the selected categories.

The team identified the challenges and began to construct a strategy wherein proven Figure measures could be designed in response to the key factors presented in the Pareto chart. How this was going to be executed required a systematic and structured process to ensure everyone was kept on track and the stages measured. A project charter was developed in line with this methodology with key stakeholders assigned to lead and deliver. The consensus of the team identified overcrowding, a lack of organisational risk assessment and management, along with reactiveness to situations were among the top three challenges Wolf 2014 [14].

Using Sirkin et al (2005) DICE Theory (illustrated in Figure 9) the team calculated their probability of success and sustainability using the basic formulae. The score was 9 which placed them in the 'win' zone.

Table 1. DICE Score.

\begin{tabular}{lllll}
\hline Formula: $\mathbf{D}+(\mathbf{2} \mathbf{2})+(\mathbf{2 x}$ C1) $+\mathbf{C 2}=\mathbf{E}$ & & & \\
\hline Duration & Integrity (Team performance) & Commitment (Management) & Commitment (local) & Effort \\
\hline$<2$ months $=1$ & Very good $=1$ & Clear and strong commitment of need $=1$ & Eager $=1$ & $<10 \%=$ additional $=1$ \\
$<2$ months $=2$ & Good $=2$ & Seem to want success $=2$ & Willing $=2$ & $10-20 \%=$ additional $=2$ \\
$4-8$ months $=3$ & Average $=3$ & Neutral $=3$ & Reluctant $=3$ & $20-40 \%=$ additional $=3$ \\
$>8$ months & Poor $=4$ & Reluctant $=4$ & Strongly reluctant $=4$ & $>40 \%=$ additional $=4$ \\
\hline
\end{tabular}

The illustration is of the overarching project charter which lays out the problems, methods used, time frames and objectives in a single, concise document.

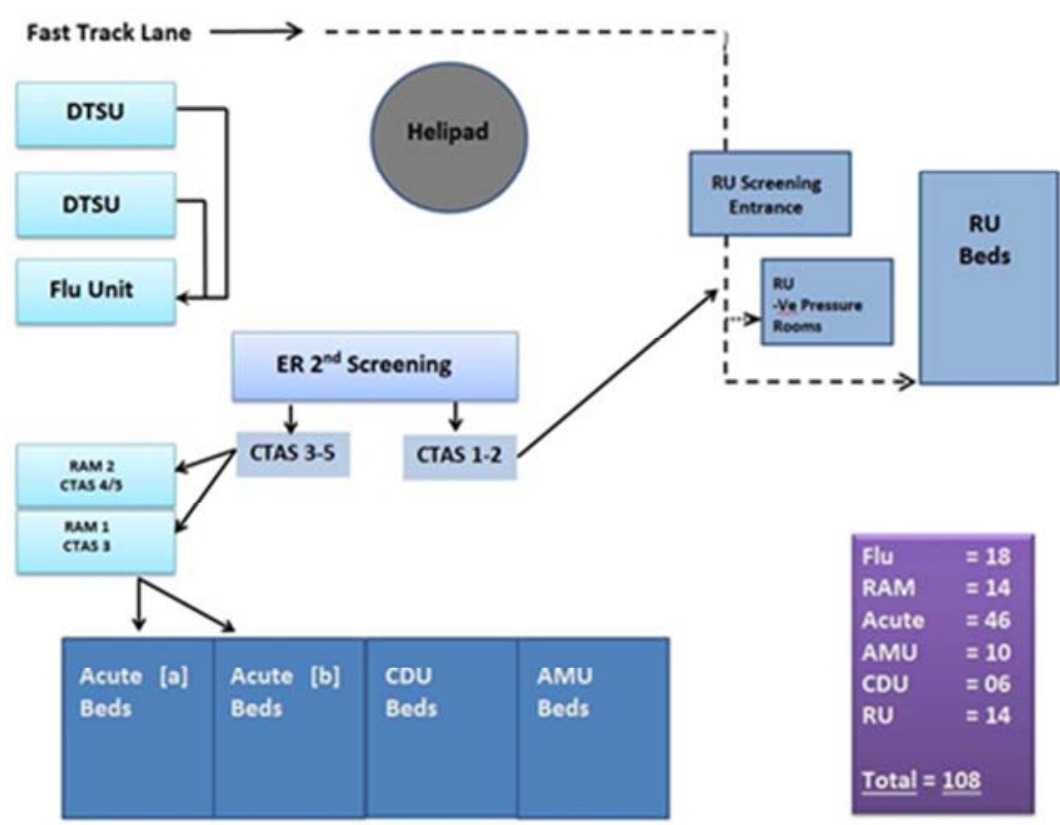

Figure 9. Caswell-Hijazi Model. 
Legend: DTU drive through screening unit. RAM (1) \& (2) rapid assessment and management areas for CTAS categories related to acuity. CTAS: Canadian Triage Acuity Scale. AMU: acute medical unit for short term cases requiring $>12$ but $<72$ hours inpatient stay. CDU: clinical decision unit where patients may require close but short term observation before either discharge or admission. Length of stay in this unit not to exceed 12 hours. RU Resuscitation Unit for CTAS 1 \& 2 cases requiring immediate management for life threatening conditions. Acute Care (a) and (b) two 24 bed areas for the management of level 3 patients that should not exceed 6 hours in the department before decision to either discharge, admit to CDU for further short term observation, transfer to AMU or in patient ward. Flu Unit where patients directed who had been identified as potential risk of MERS-Cov infected using ARI screening tool. Secondary lane in DRU for ambulances would fast track through to secondary RU screening area located at the side entrance created for the RU. Rapid screening would be performed by staff of any acuity CTAS 1 and 2 patients and any suspected case taken directly to a separate negative pressure area created at the far end of the RU and physically divided with tempered glass.

Primary screening units were built at the entrance to the ED. Designed to screen any adult patient for signs of Acute Respiratory Infection (ARI) in accordance to the case definition of MERS-Cov provided by the Ministry of Health and endorsed by the CDC and WHO. Any patient who triggered as a suspected case would receive a red stamp screening form and directed to the Flu Unit located directly to the right of the (DTSU) approximately 20 meters away with separately allocated parking spaces. The unit comprised of 16 single negative pressure rooms and 2 negative pressure resuscitation rooms located immediately inside the unit to safely manage any patients who might deteriorate following arrival to the area meant for the management of Canadian Triage and Acuity Scale (CTAS) level 3-5 cases.

On arrival to the Flu Unit relatives were asked to wait inside the assigned waiting areas. Patients provided with mask and hand hygiene gel dispensed to them with instructions as to its use, the purpose and the importance of marinating the mask. The objective was to transit the patient directly into a room without delays and vital signs performed immediately. Patient seen within 15 minutes by a physician and plan made in relation to treatment and any diagnostic investigations required. The patient would remain in the unit until a decision was made for admission. Those with a high index of suspicion, transported via ambulance to a designated ward inside the main hospital avoiding contact with others. Patients with a low index of suspicion but who had been swabbed were discharged home with clear instructions for home isolation and education to patient and family was completed along with written advice. The IPC team would track any such patient in relation to the results and take necessary actions such as contacting the patient with the results and be available $24 / 7$ for advice as a 'hot line' to answer any questions or concerns.

The Flu Unit had its assigned staff as well as housekeepers.
It was imperative that this team worked closely adhering to the strictest Infection Prevention and Control (IPC) precautions and surveillance of each other's practice was essential. Every room was cleaned immediately following the patient's discharge with a turnaround time of 30 minutes.

Any ambulance with severely ill patients or private cars who stated their patients were very sick would be sent directly to the resuscitation unit entrance where an ARI rapid screening would be undertaken by staff assigned to the area $24 / 7$. Any CTAS level $1 / 2$ cases who triggered as suspected ARI would enter the redesigned resuscitation unit directly into one of 4 negative pressure rooms located at the far end of the unit separated by a clear glass wall. Staff assigned to this sub-unit would work as buddies, maintaining strict surveillance in relation to the adherence to IPC procedures. For patients considered as having a high index of suspicion related to their clinical presentation and symptoms would be escalated immediately to the department of epidemiology, IPC and laboratory.

Upon arrival to the main ED entrance, a secondary screening area was established to capture any patients who may have gained access via another route or who may have walked in past the DTSU.

In an attempt to reduce the risk of access to the ED via alternative routes and in order to minimize unnecessary traffic in the area, the main hospital access doors were locked down and a guard stationed at the entrance 24/7. Only those who had been issued with a swipe access card could gain entry. This proved extremely effective in preventing patents and staff from using the department as a short cut route. The department had effectively achieved a lock down and lock- out capability which supported a significant reduction in footfall.

Any patients who triggered as a 'red' and triaged as level 3-5 were redirected to the Flu Unit and phone call made to the desk therein to expect the patient.

All other patients would be directed to the triage area located to the left of the secondary screening area for comprehensive triage following Across the Room Assessment (ATRA).

Patients of CTAS 1 or 2 were immediately taken to the resuscitation unit. Level 3 cases seen and assessed in Rapid Assessment and Management (RAM) 1 with the target time of initial assessment by a physician within 15 minutes of arrival. The nurse and physician working together to record vital signs, conduct a preliminary assessment and plan for diagnostic work up or treatment. The patient would be taken directly to Acute Care (a) or (b) both areas managed synergistically as a mirror image of the other. The objective was to complete the work up and management of the plan within (4) hours from arrival to a final decision and a further (2) hours allocated to admitting team and bed management to transfer the patient out of the department. For patients requiring hospital admission the policy clearly stated that the admitting team had a further 2 hours from time of referral from ED to transferring the patient out of the department and into a sui Figure bed. The decision would be to discharge home, refer for admission, to manage in the Clinical 
Decision Unit (CDU) for cases fitting the admission criteria, or to be admitted to the Acute Medical Unit (AMU) for cases, that again met the admission criteria for whom a rapid access to diagnostic procedures and or management e.g. up to 72 hours antibiotic administration and discharge.

There had been some initial resistance and challenges related to this performance target time but during a series of PDSA cycles, the decision was made to accommodate key personnel from bed management to work from within the department alongside the nurse assigned per shift to manage internal patient flow, this was the role of the Nurse Patient Flow coordinator who orchestrated movement and was vigilant of any delays that would be highlighted and escalated.

Patients triaged as CTAS 4-5 were sent immediately to RAM 2 where the target time would be seen by the physician within 30 minutes. The same model of care would be applied in that the nurse and physician would work in partnership to create a 'one stop shop' of assessment and management before discharge. Many of these patients required reassurance and advice and were provided with appointments to attend their local primary care clinics. Initial challenges for this area had been in the re-presentation of patients within 24 hours for similar issues. This matter had been examined as part of the Key Performance Indicators (KPI's), and a monthly analysis of trends made. The CTAS levels had not changed but following dialogue with patients who returned to the department, they had experienced difficulties in getting appointments that were allocated at a sui Figure time and date. It is worthy to note that this is a culture of varied levels of education and understanding. The males of the family is the only one who can drive and many stricter families do not allow females to travel via taxi without being accompanied by male family members. This meant that males had to arrange time off work to transport and wait for family members. In many ways the minimal waiting time the department had achieved meant that they had become the victims of their own success as word soon spread that this was an area one could visit without delays and where patients are seen and treated by consultants and experienced personnel.

The team liaised with primary care centers and the department was issued with a set number of protected appointment slots daily, including weekends. Primary care clinics operating from 09:00-24:00 as it must be understood that culturally, this is a primarily nocturnal culture. This is a characteristic reflected in the increased activity of the adult and pediatric emergency departments at night. This was particularly evident during the Eid Holidays when adults and older children fast from sunrise until sunset for a 2 week period. Following the breaking of the fast, there is prayer at local mosques and then feasts. There is an increase in the number of diabetics who attend the department as well as the number of motor vehicle accidents frequently caused by tired and hungry drivers.

Acutely sick patients managed in the resuscitation unit are assessed based upon their clinical stability before any transfer is made and therefore are exempt from the 6 hour target as required.
The robust push/pull model was introduced with the prime objective of preventing overcrowding from occurring the ED and ensuring that patients receive optimal care and management within the appropriate clinical area related to their physical needs Ordesson et al 2011 [15]. The moths that followed meant the department and the organisational systems in place were being tested and scrutinized by not only the executive leadership team but external organisations such as the Ministry of Health and CDC who made repeated unannounced visits at night and weekends.

As patients waiting inpatient beds began to face delays exceeding 6 hours a new approach was required to proactively deal with the whole hospital issues. Daily team meetings were held between bed management, ED and representatives from each division. An assessment of the demands from the ED, elective patients and demands on inpatient beds was made. Any delays with complex discharges discussed along with action plans in place to deal with problem areas. It was acknowledged that the ED was indeed the organisation's thermometer for pressure, capacity and risk.

\section{References}

[1] Zaid, M. Abdullah, A. Rafaat, A. Suben, Y. Malak, A. Alimuddin, Z. Al-Tawfiq, J. A. Christian, D. Ali, A. Eskild, P. 2014. Middle Eastern Respiratory Corona Virus, MERSCovConclusions from the $2^{\text {nd }}$ Scientific.

[2] Sirkin, HL. Keenan, P. Jackson, A. The Hard Side of Change Management 2005. Harvard Business Review.

[3] McCaig and Nawar 2006. Variation in Emergency Department Wait Times for Children by Race/Ethnicity and Payment Source, Frequent Overcrowding in U.S. Emergency Departments ACADEMIC EMERGENCY MEDICINE February 2001, Volume 8, Number 2151.

[4] Nadler, D. A., \& Tushman, M. L. 1977. A diagnostic model for organization behavior. In J. R. Hackman, E. E. Lawler, \& L. W. Porter (Eds.), Perspectives on behavior in organizations: 85-100. New York: McGraw-Hill.

[5] David I Ben-Tovim, Jane E Bassham, Denise M Bennett, Melissa L Dougherty, Margaret A Martin, Susan J O’Neill, Jackie L Sincock and Michael G Szwarcbord. Redesigning care at the Flinders Medical Centre: clinical process redesign using "lean thinking." Med J Aust 2008; 188 (6 Suppl): S27.

[6] Sahin M. K. Aker S. Tuncel E. K. Knowledge, Attitudes and Practices Concerning Middle East Respiratory Syndrome Among Umrah and Hajj Pilgrims in Samsun, Turkey 2015. Advisory Board Meeting of the WHO Collaborating Centre for Mass Gathering Medicine, Riyadh. International Journal of Infectious Disease. Volume 24, pp 51-53.

[7] Armony M. Israelit S. Mandelbaum A. Marmer Y. N. Tseytlin Y. Yom-Tov G. B. On Patient Flow in Hospitals: a data-based queueing science perspective. Stochastic Systems 2015 volume5 \#1: 146-19.

[8] Singer, A. J. Thode, H. C. Viccellio, P. Pines, J. M. The Association between Length of Emergency Department Boarding and Mortality. Academic Emergency Medicine $13^{\text {th }}$ December 2011. 
[9] Beveridge, R. Clarke, B. Janes, L. Savage, N. Thompson, J. Dodd, G. Murray, H. Nijssen-Jordan, C. Warren, D. Vandebencoeur, A. Implementation Guidelines for The Canadian Emergency Department Triage and Acuity Scale (CTAS). Version 16. November 1998.

[10] Scott, I. Vaughan, L. Bell, D. International Journal of quality Health Care (2009) 21 (6): 397-407.

[11] Hassan, T. B. Clinical decision units in the emergency department: old concepts, new paradigm, and refining gate keeping. Emergency Medical Journal 2003: 20: 123-125.

[12] Pearson, S. D. Goulart- Fisher, D. Lee, T. H. Clinical pathways as a strategy for improving care: problems and potential. Ann. International Med. 1995, 123: 941-8.
[13] Scoville, R. Little, K. Comparing Lean and Quality Improvement, Cambridge, Massachusses Institute for Healthcare Improvement. 2014.

[14] Wolf, L. 2014 Research as Problem Solving: Theoretical Frameworks as Tools. Journal of Emergency Nursing Volume 41. Issue $1 \mathrm{p}$ 83-85.

[15] Ordensson, S. Johnsson, H. Rognes, J. Lind, L. Goransson, K. Ehrenberg, A. Asplund, K. Castren, M. Farrohkina, N. 2011. A systematic review of triage-related interventions to improve patient flow in emergency departments. Scandinavian Journal of Trauma, Resuscitation and Emergency Medicine. 19: 43. 